\title{
SEMI-STRUCTURED DESIGN AND PROBLEM-BASED EXPERIENTIAL LEARNING IN A FIRST-YEAR BIOMEDICAL ENGINEERING LABORATORY COURSE
}

\author{
Gabrielle Lam $^{1,2}$, Navjot Gill ${ }^{1}$, Roza Ghaemi ${ }^{1}$ \\ ${ }^{1}$ School of Biomedical Engineering, ${ }^{2}$ Department of Materials Engineering \\ University of British Columbia, Canada \\ Corresponding author: gabrielle.lam@ubc.ca
}

\begin{abstract}
A first-year biomedical engineering laboratory course was created using a problem-based approach, introducing students to the interplay of engineering design and biology in biomedical engineering. Open-ended problems were central to each laboratory module, and were designed to enhance students' development of high-order learning skills. Although the value of problem-based learning in engineering laboratory courses has been recognized, its implementation in the first-year engineering program presents unique challenges for students who are unfamiliar with the unstructured approach and who are in their early stages of developing discipline-specific knowledge. Immediate feedback scaffolding strategies, including interactive pre-laboratory assessments and team-based quizzes, were implemented in the second iteration of the course to support students' achievement of learning outcomes, and to improve their perception of their learning experience in a problem-based laboratory. According to preliminary survey results, students attributed the value of problem-based laboratory activities to the acquisition of technical skills, engineering design skills, as well as the development of self-directed ability, and a growth mindset. Results from the second iteration of the course also revealed that students perceived the interactive pre-laboratory assessments and team-based quizzes to be highly valuable. Taken together, our study thus far has underlined the importance of immediate feedback as an effective scaffolding strategy for supporting semi-structured experiential learning in a firstyear engineering laboratory course.
\end{abstract}

Keywords: Problem-based learning, experiential learning, engineering design, biomedical engineering, laboratory course, first-year engineering

\section{INTRODUCTION}

\subsection{Motivation for a problem-based approach}

The Biomedical Engineering Laboratory is a firstyear laboratory course that was created as part of the new undergraduate biomedical engineering program at the School of Biomedical Engineering, University of British Columbia (UBC). The course was offered to its first student cohort in January 2019, and is taken by engineering undergraduate students admitted to the pre-biomedical engineering program in their first-year at UBC. The course was designed to use experiential learning to introduce students to the interdisciplinary nature of biomedical engineering, as well as the interplay between engineering design and biology. The intent is that, through effectively designed experiential learning opportunities, students may develop high-order learning skills as early as in their firstyear undergraduate program. These skills can subsequently equip students to contribute to the interdisciplinary challenges in the biomedical engineering field in their future careers.

Laboratory courses are an integral part of many undergraduate engineering programs. However, its value to student learning has been widely discussed. Some studies have shown that the effect of laboratory courses on enhancing student learning is largely dependent upon its implementation [1,2]. Holmes and Wieman, for example, evaluated the efficacy of experiential learning in introductory physics courses across three American universities [2]. Based on their investigation, students who enrolled in an optional physics laboratory course did not see significant improvement in their achievement of learning outcomes when compared to students who did not enroll in the optional laboratory course. This is a perplexing finding, especially when considering that laboratory courses are typically resource- and timeintensive.

Traditional laboratory courses are technique-driven and involve highly structured activities. In these activities, students are often focused on completing meticulous procedures, in search of a "correct" answer. This approach can encourage students to take on a passive role in learning, ultimately leading to surface learning [3]. Unfortunately, the outcome is often contrary to the intended effect, with students exercising little knowledge synthesis and development of high-order learning skills. 
More recently, an open-ended problem-based approach has been implemented in science and engineering laboratory courses, with the intent of improving student motivation and learning [4-7]. Problem-based learning (PBL) was originally developed and used at the McMaster Medical School in Canada [8]. Unlike highly structured approaches, PBL relies on the use of open-ended, illstructured and complex problems which often require students to evaluate multiple solution paths [9], thereby enhancing self-directed learning and development of critical thinking skills [10]. Based on a meta-analysis of literature conducted by Gijbels et al. [11], compared to conventional formats like lecture-based learning, PBL improves students' understanding of the interconnection between concepts and their ability to apply these concepts for problem-solving.

This problem-based approach has been adapted to undergraduate engineering laboratory courses. Its effects on students' achievement of learning outcomes and their impressions of their learning experience were compared to that of structured course formats. Hartman and Gindy, for example, re-designed a Civil and Environmental Engineering laboratory course using a problem-based approach, with the implementation of open-ended, unstructured and multidisciplinary projects [6]. Compared to students in the control group (traditional instructor-led approach), those in the experimental group (PBL-centric approach) perceived that they developed a greater ability to design experiments, analyze data, design engineering systems, and evaluate outcomes and solutions. At the same time, these students also demonstrated greater apprehension about the lack of structure in the course, expressing a greater desire for guidance and instructions, compared to students in the control group. Newstetter et al. compared students' achievement of learning outcomes in a biomedical engineering laboratory course using a technique-driven versus problem-based approach [7]. According to results from an end-of-term survey, students in the experimental (PBL) group were more confident in identifying critical problem features and solutions, as well as their ability to learn from failure. Interestingly, these students also perceived themselves as less competent in executing lab techniques and using lab equipment, compared to students in the control (techniques-driven) group. Yet, results from an end-of-term comprehension test showed that students exposed to the PBL approach were in fact better able to apply newly gained knowledge in the areas of definitions, laboratory instrumentation and experimental design.

\subsection{Scaffolding strategies}

Despite the advantages associated with a problembased approach, the unstructured nature of PBL can induce significant stress and frustration in students. This was evident in student responses to an end-of-term survey in
Hartman and Gindy's study of a PBL versus instructor-led approach to a Civil and Environmental Engineering laboratory course [6]. Similar observations motivated Newstetter et al. to propose further constraining openended laboratory activities in future iterations of a Biomedical Engineering laboratory course [7]. Moreover, Gijbel and colleague's meta-analysis of PBL indicates no obvious benefit in improving knowledge transfer, when compared to conventional (such as lecture-based) approaches [11].

Taken together, although PBL can improve student learning and development of critical thinking in laboratory courses, it can also pose unique challenges in the first-year setting. Specifically, first-year engineering students likely had little prior exposure to unstructured problems, and are also in their early stages of building discipline-specific knowledge and skills. The effect of these factors on students' learning experience and ability to achieve learning outcomes must be considered when designing and implementing PBL activities in first-year courses.

To this end, scaffolds are instructional components or tools that have been used to provide additional structure in PBL $[12,13]$. According to Schmidt et al., scaffolds reduce students' cognitive effort associated with learning new material, allowing students to focus on problem-solving instead [14]. Moreover, the use of scaffolds can be progressively reduced over the duration of a course or program, as students become more familiar with a problem-based approach [15].

Clark and Mahboobin studied the effect of scaffolding on student performance in a PBL bioengineering laboratory course [16]. The scaffolding strategies used included: 1) exercises that guided students in problem decomposition, 2) worked examples, and 3) timely instructor feedback to progress reports. Compared to a non-scaffolded iteration of the course, these strategies improved student scores on open-ended projects. Likewise, Frank et al. found that provision of formative feedback in a first-year engineering design course improved student performance in complex problem solving over the duration of the course [17]. Proper scaffolding of PBL is therefore expected to support students' development of high-order learning skills, while improving their impressions of their learning experience.

\subsection{Significance of results}

The objective of this study is to investigate the effect of immediate feedback scaffolding strategies on: 1) student perception of their learning experience and 2) student performance in problem-based laboratory activities. While various studies have demonstrated the value of implementing a problem-based approach in engineering laboratory courses, the unstructured nature of PBL can present unique challenges to first-year undergraduate students. This is particularly relevant in the context of a 
first-year biomedical engineering laboratory, in which students are introduced to a broad range of new disciplinespecific problems, concepts and techniques. Delivery of timely formative feedback from the instructor, such as through in-class verbal feedback or written feedback on formative assessments, can contribute significantly to student performance and their perceived preparedness to handle open-ended problems. Practically, however, this is challenging to implement in a large-class setting (77 students were enrolled in the course in January 2020, and steady-state enrolment is expected to be 110 students). The results of this study can therefore shed light on the role of immediate feedback strategies as a method of scaffolding problem-based laboratory activities for a large first-year engineering laboratory course. From here on in, this will be referred to as a semi-structured problem-based approach.

\section{METHODS}

\subsection{Course design and structure}

Introduction to Biomedical Engineering Laboratory is comprised of four laboratory modules, each associated with one of the four technical streams of the biomedical engineering undergraduate program at UBC. Students are assigned to teams from the start of the course, and continue to work within this same team throughout the duration of the course. During the lecture component of the course, students are introduced to the biological concepts that are relevant to each module-specific project.

Beginning from the first iteration of the course, a problem-based approach was used in the design of all laboratory modules. Open-ended laboratory projects required students to either: 1) design an experiment to answer a research question, or 2) evaluate possible solutions to a design problem.

In the first iteration of the course (January 2019), students individually completed a pre-laboratory assessment prior to beginning each laboratory module. Prelaboratory assessments took the form of electronic submissions of students' answers to short-answer questions. At the end of each laboratory module, students worked within their teams to complete a project submission.

In the second iteration of the course (January 2020), interactive pre-laboratory assessments were created and implemented to provide students with immediate formative feedback. Interactive online pre-laboratory assessments were comprised of interactive components (such as clickable information buttons and videos), and interspersed questions with immediate feedback (solutions). Assessment questions were delivered in the form of multiple choice, fill-in-the-blank, and hotspot selection formats. Most importantly, the online interactive prelaboratory assessments provided students automated and immediate feedback as they progressed through learning new concepts and techniques. Additionally, during the second iteration of the course, a new team-based quiz was implemented at the start of each laboratory module. The Immediate Feedback Assessment Technique (IF-AT) system was used to provide student teams with immediate feedback, and to support team-based learning in the laboratory [18]. A last course component that was newly created in the second iteration of the course was a mini design project. The open-ended mini design project was implemented at the end of the course, and was designed to build upon students' knowledge and skills developed from all previous laboratory modules.

\subsection{Study structure}

In the second iteration of the course, the immediate feedback scaffolding strategies - interactive online prelaboratory assessments and team-based quizzes - were implemented in two of four laboratory modules (experimental groups: Cell bioengineering and Bioinformatics modules). These were introduced alongside two laboratory modules without immediate feedback scaffolding strategies (control groups: Biomaterials and biomechanics, and Electrical systems and signals modules). The study was designed using an ABAB structure, according to Figure 1 below. This allowed students within the same cohort to make direct comparisons between modules with immediate feedback scaffolding strategies (experimental) versus those without (control).

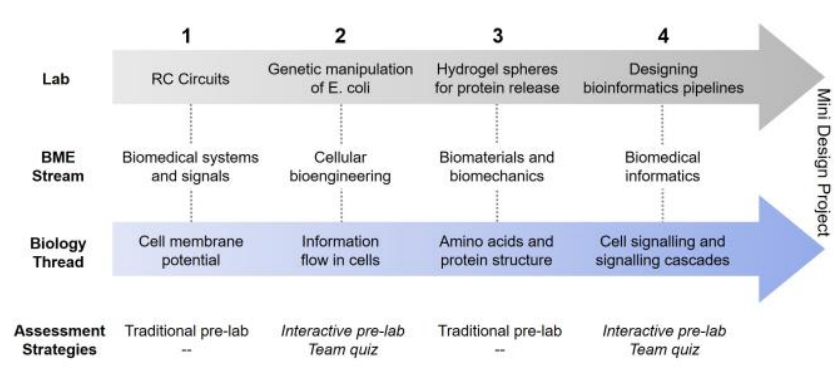

Figure 1. Schematic of the Biomedical Engineering Laboratory course structure, with old and new laboratory curriculum arranged in an ABAB structure.

\subsection{Module-specific surveys}

In both the first and second iteration of the course (January 2019 and January 2020), module-specific surveys were administered at the end of each laboratory module to gauge students' perception of the level of difficulty, relevance and value of each lab activity. In the second iteration of the course, surveys for modules in the experimental group also contained questions to gauge student perception of the value of interactive online prelaboratory assessments and team-based quizzes in improving student preparedness. Survey completion was 
voluntary and student responses were recorded anonymously through Qualtrics. All module-specific surveys also contained two open-ended questions which prompted students to describe:

- Question 1: Something that [they] gained from [the] module (technical or non-technical) that [they] will carry into the rest of the course, or [their] degree

- Question 2: One way (or more) [the] module could be improved or changed

Descriptive responses from surveys collected from the first iteration of the course were analyzed qualitatively using an open-coding method. These codes were subsequently categorized into larger sub-themes and themes. Analysis of descriptive responses from surveys collected from the second iteration of the course is currently ongoing.

\section{RESULTS}

Qualitative analysis was conducted on student responses to descriptive Questions 1 and 2 from data obtained in the first iteration of the course (January 2019). Analyses of student responses to the first survey prompt (Question 1) "Something that I gained from this module (technical or non-technical) that I will carry into the rest of the course, or my degree" revealed six major themes in the data. The six major themes included: domain knowledge, technical skills, practice of engineering design, growth mindset, self-directed ability and interpersonal skills. These thematic categories, their associated sub-themes, codes and select examples of student responses are summarized in Appendix Table 1.

Subsequently, the frequencies of student responses associated with each major theme were quantified. Results show that, when describing the value derived from each laboratory module, students referred most frequently to the acquisition of technical skills. At the same time, students also recognized the value of practicing engineering design, and of developing self-directed ability, albeit to a lesser extent. The frequencies of student responses associated with each of the six thematic categories, according to laboratory module, are summarized in Figure 2.

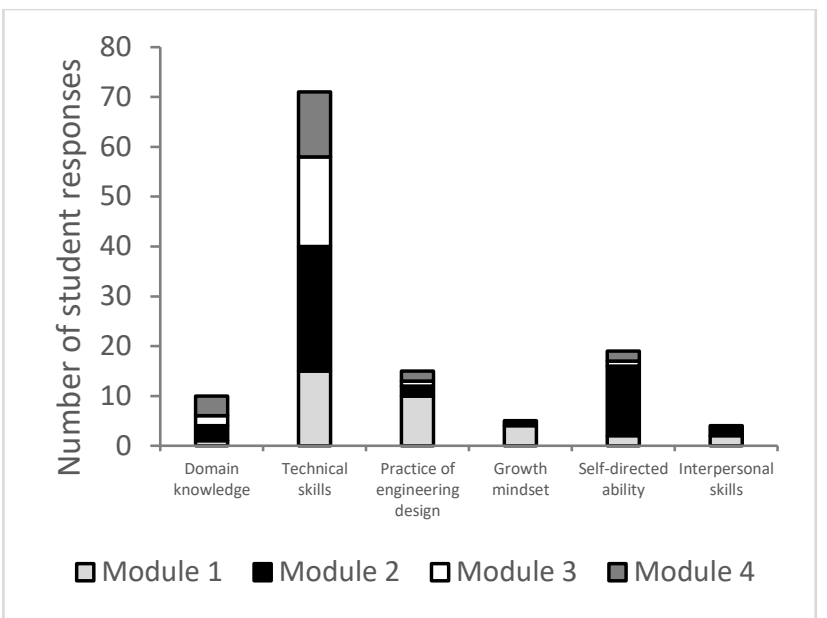

Figure 2. Number of student responses associated with each major theme in survey Question 1.

Apart from describing the importance of gaining technical skills, students most frequently described the value of developing self-directed ability in their laboratory modules. Most of these responses were collected from the Module 2 survey. Surprisingly, this activity was also the most challenging, as reflected by lowest student performance on the lab assessment (data not shown).

Qualitative analysis of student responses to the survey prompt (Question 2) "One way (or more) this module could be improved or changed" revealed four major themes in the data. Student responses described improvements to instructional materials, teaching practices, and time allocation. The sub-themes, codes and select student examples for each thematic category are presented in Appendix Table 2.

Considering data from all module surveys collectively, the greatest number of student responses were related to improving instructional guidance in lecture (teaching practice) and in instructional materials (laboratory manuals). The overwhelming majority of these responses were associated with the Module 2 activity, which was also the most challenging activity, indicated by lowest student performance on assessments. Figure 3 shows the frequency of student responses related to each of the four thematic categories, according to laboratory module. 


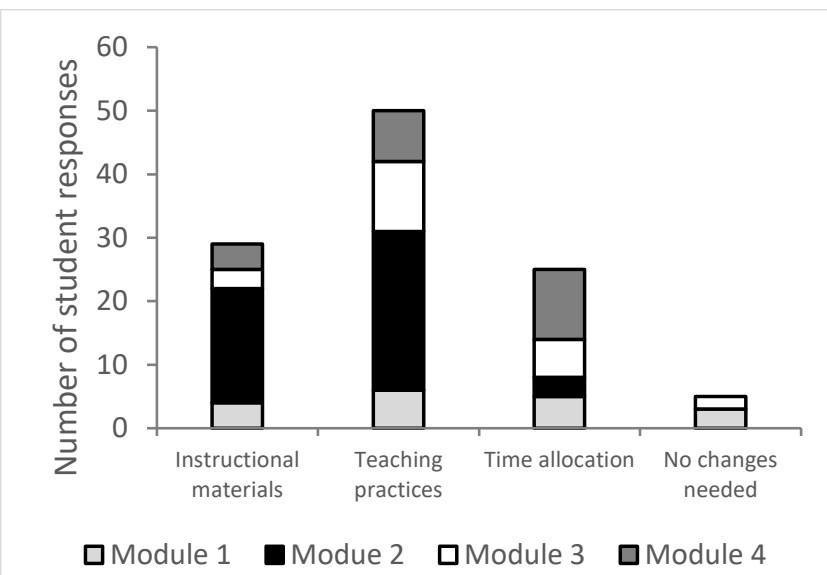

Figure 3. Number of student responses associated with each of major themes in survey Question 2.

Student responses to survey questions were also analyzed to investigate their perceived value of the immediate feedback scaffolding strategies introduced during the second iteration of the course. The interactive online pre-laboratory assessments and team-based quizzes were implemented in Module 2 (Cellular bioengineering module) and Module 4 (Bioinformatics module). By contrast, Module 1 (Systems and signals) and Module 3 (Biomaterials and biomechanics) did not involve the use of these immediate feedback scaffolding strategies; instead, students were only required to submit a "traditional" prelaboratory assessment (Figure 1). As described previously, an $\mathrm{ABAB}$ study structure was used so that students within the same cohort could directly compare their learning experience with versus without immediate feedback scaffolding strategies.

Results from student responses to the survey question "The interactive pre-lab was more effective in preparing me for the lab activity, compared to the traditional (document-style) pre-lab" are summarized in Figure 4. The survey question was specific to laboratory modules from the experimental group (Modules 2 and 4). For both modules in the experimental group, an overwhelming majority of students strongly agree that the interactive prelaboratory assessment was more effective than the traditional format (70\% of student responses, or 32 of 46 responses in the Module 2 survey; $69 \%$ of student responses, or 9 of 13 responses in the Module 4 survey).

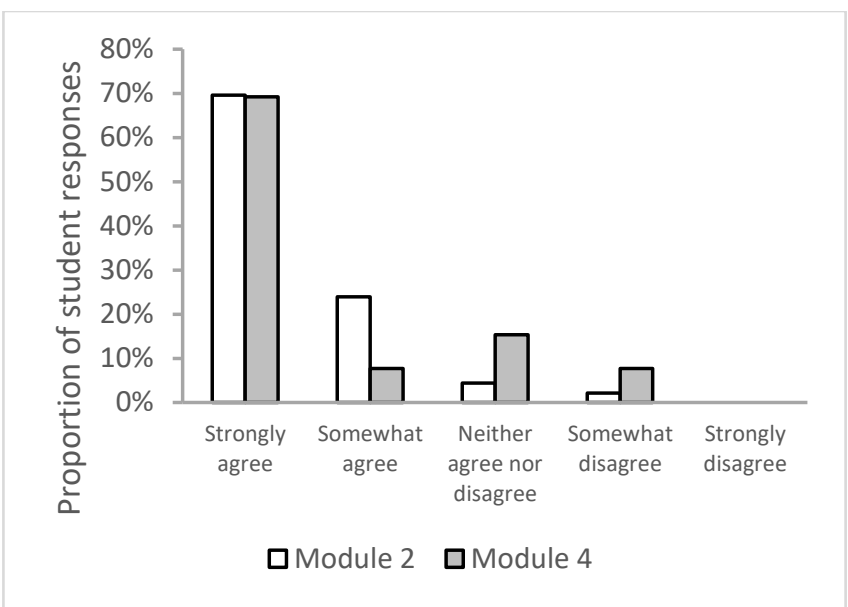

Figure 4. Student perception of the value of the interactive versus traditional pre-laboratory assessment.

In order to identify the specific aspects of the interactive pre-laboratory assessment that were perceived to be valuable, students were prompted to evaluate the value of: 1) the interactive content (clickable buttons and content), 2) interspersed questions, and 3) immediate feedback to questions. Results from Module 2 and Module 4 surveys are summarized in Figure 5 below.

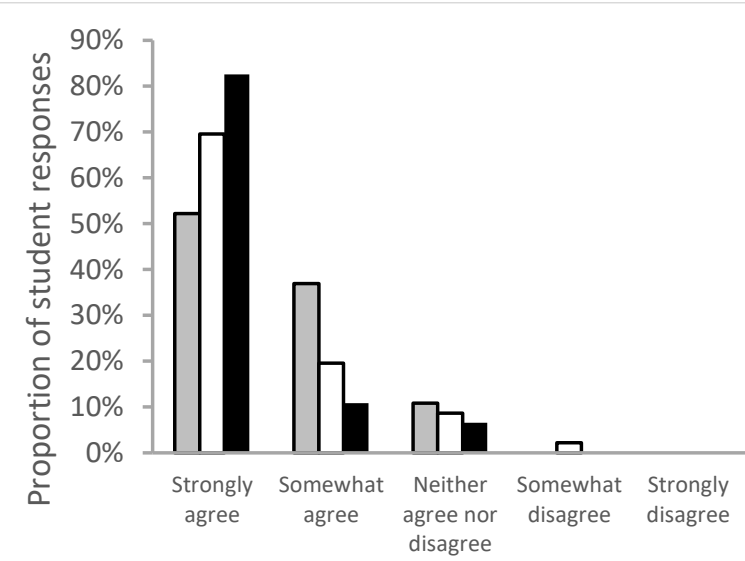

口Interactive content

口Interspersed questions

- Immediate feedback to question solutions 


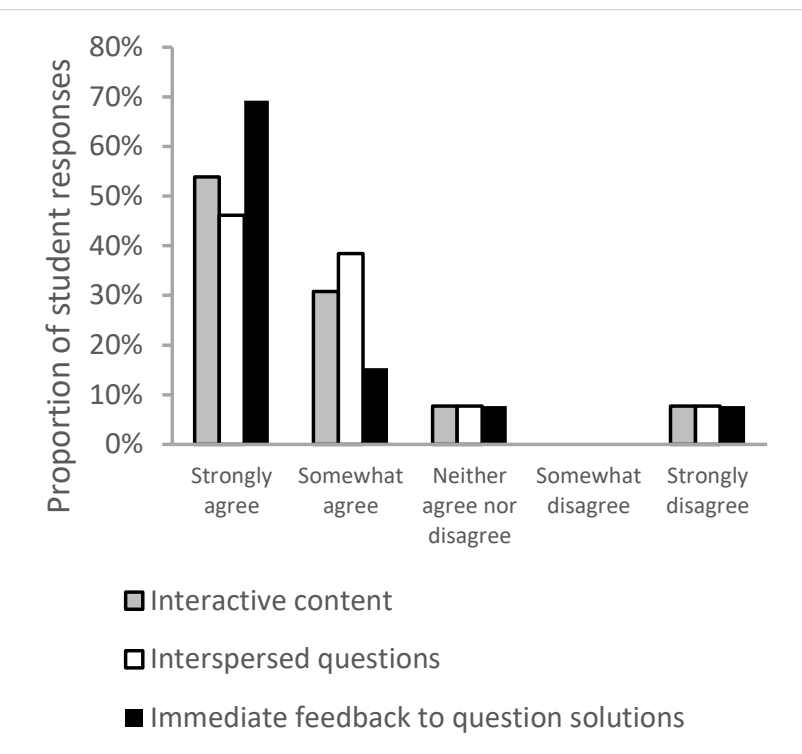

Figure 5. Student perception of the value of interactive content, interspersed questions, and immediacy of feedback in the interactive pre-laboratory assessments from the Module 2 survey (top) and Module 4 survey (bottom).

According to results, all three aspects of the interactive pre-laboratory assessment were perceived to be valuable. In particular, the immediacy of feedback to question solutions was perceived to be the most valuable aspect of the interactive pre-laboratory assessment, with $83 \%$ of student responses (38 of 46 responses) strongly agreeing to the statement for Module 2, and 69\% of student responses (9 of 13 responses) strongly agreeing to the statement for Module 4.

Results from student responses to the survey question 'The team quiz was effective in bringing all team members' understanding of concepts to a similar level prior to the lab activity" are presented in Figure 6. The majority of students answered favorably (either "somewhat agree" or "strongly agree"). Whereas $36 \%$ of student responses (16 of 45 responses) strongly agreed to the statement for Module 2, $46 \%$ of student responses (6 of 13 responses) strongly agreed to the statement for Module 4.

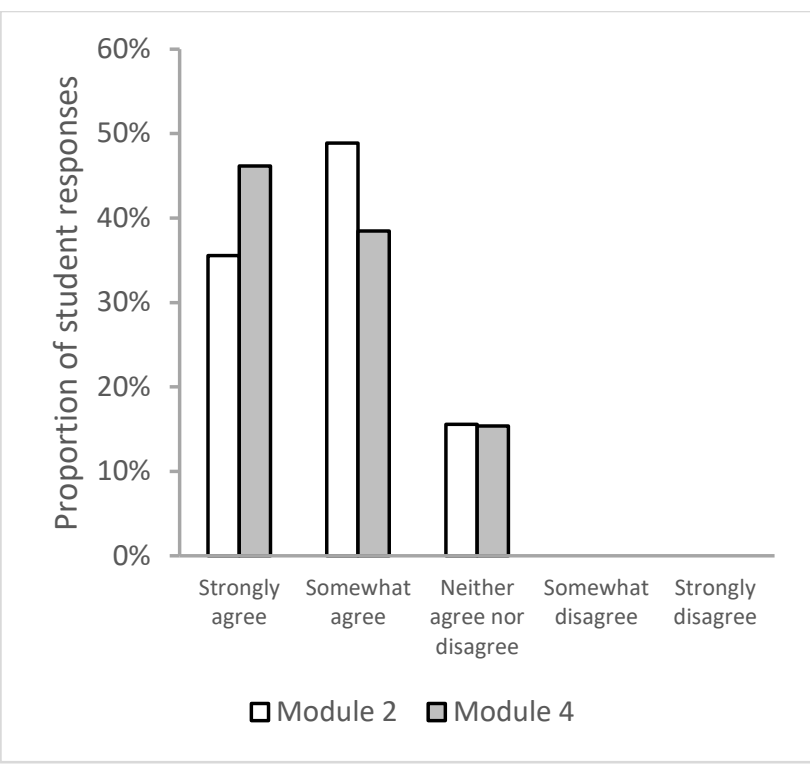

Figure 6. Student perception of the value of the teambased IF-AT quiz.

\section{DISCUSSION}

Qualitative results from student responses to two open-ended survey questions obtained during the first iteration of the course revealed that students primarily related the value of their experiential learning to the acquisition of technical skills. This is not a surprising observation, given that the course introduces students to new and specialized equipment, from oscilloscopes, to volumetric pipettes, to spectrophotometers. That said, student responses also revealed a recognition of the value of practicing engineering design, of developing nontechnical skills, such as those associated with a growth mindset and self-directed ability.

Interestingly, the laboratory module that was associated with the greatest frequency of student responses related to the value of "self-directed ability" was also the most challenging laboratory module (Module 2), as indicated by lowest overall student performance (data not shown). Compared to survey responses in all other modules, student responses to survey questions from Module 2 showed the highest frequency of student reflection upon the value of intrinsic motivation, autonomy and self-regulation. At the same time, it also corresponded to the highest frequency of student reflection on the need for greater guidance in teaching practices (in lectures and laboratories) and detailed instructions from teaching materials (lab manuals). This finding is consistent with other studies $[6,7]$, in which students reflected a desire for more detailed instructions in PBL courses.

Taken together, these preliminary results suggest that a problem-based approach to a first-year engineering 
laboratory curriculum can stimulate student appreciation and reflection of higher-order learning skills, including those related to engineering design, growth mindset, selfdirected ability and interpersonal skills. However, implementation of appropriate scaffolding strategies has the potential to further improve students' learning experience, presumably by reducing students' apprehension and frustration regarding open-ended problems.

For this reason, two scaffolding strategies were newly implemented in the second iteration of the course (January 2020). The interactive online pre-laboratory assessments and team-based quizzes (IF-AT format) were designed and implemented to provide students with immediate feedback on formative assessments. Whereas the interactive online pre-laboratory assessments were completed individually, the team-based quiz was completed as a group prior to the start of a laboratory module. Student perception of the value of interactive online pre-laboratory assessments was overwhelmingly favorable, with around $70 \%$ of students strongly agreeing that the interactive online pre-laboratory assessment was more valuable that the traditional prelaboratory assessment format. The three main aspects of the interactive online pre-laboratory assessment were perceived to be valuable, including the use of interactive content, inclusion of interspersed questions, and provision of immediate feedback to questions. Of the three aspects, immediate feedback to questions was associated with the greatest frequency of highly favorable student responses ("strongly agree").

In addition to introducing the interactive online prelaboratory assessments, a team-based IF-AT quiz was newly implemented in the second iteration of the course (January 2020). Using the IF-AT system, students worked with their team members to answer a series of multiplechoice questions prior to the start of a laboratory module. The system provided immediate feedback to the team, such that they could continue to discuss their solution until the problem was correctly resolved. The IF-AT quiz was implemented to bring all team members' understanding of concepts to a similar level prior to the lab activity, by encouraging students to discuss and compare solutions with their team members. Overall, students perceived the team-based quiz to be valuable, with an overwhelming majority of students reflecting favorably ("strongly agree" or "somewhat agree") about its effect on normalizing team members' understanding of laboratory concepts.

Based on these initial analyses, immediate feedback to formative assessments _ whether pre-laboratory assessments or team-based quizzes - are promising scaffolding strategies for the first-year laboratory course. Moving forward, these strategies will be designed for the remaining laboratory modules and fully implemented during the third iteration of the course (January 2021). Student performance on course assessments will also be analyzed to provide insight on the effect of scaffolding strategies on students' achievement of learning outcomes. Focus group meetings will be conducted to investigate any trends revealed from survey results, and used to inform further development of the course.

\section{Acknowledgements}

We would like to thank Mathurada Jullamon, Paulina Semenec, Adriana Briseno-Garzon and Trish Varao-Sousa for their support and advice on evaluation aspects of the project. We also thank the biomedical engineering undergraduate students who have offered their time to provide thorough feedback.

\section{References}

[1] L.D. Feisel and A.J. Rosa, "The Role of the Laboratory in Undergraduate Engineering Education," J. Eng. Edu., vol. 94, no. 1, p 121-130, 2005.

[2] N.G. Holmes and C.E. Wieman, "Introductory physics labs: We can do better," Physics Today, vol. 71, no. 1, pp. 38-45, 2018.

[3] C. Major and B. Palmer, "Assessing the Effectiveness of Problem-Based Learning in Higher Education: Lessons from the Literature." Academic Exchange Quarterly, vol. 5, 2001.

[4] C. Lee, J. Su, K. Lin, J. Chang, and G. Lin, "A projectbased laboratory for learning embedded system design with industry support," IEEE Trans. Edu., vol. 53, no. 2, pp. 173-181, 2010.

[5] R.H. Chu, D.D. Lu and S. Sathiakumar, "Project-based lab teaching for power electronics and drives," IEEE Trans. Edu., vol. 51, no. 1, pp. 108-113, 2008.

[6] D. J. Hartman and M. Gindy, "Comparison of lectureand problem-based learning styles in an engineering laboratory" Transportation Research Record: Journal of the Transportation Research Board, No. 2199, p. 9 17, 2010.

[7] W.C. Newstetter, E. Behravesh, N.J. Nersessian, B.B. Fasse, "Design Principles for Problem-Driven Learning Laboratories in Biomedical Engineering Education" Annals of Biomedical Engineering, Vol. 38, No. 10, p. 3257-3267, 2010.

[8] P. Finucane, S.M. Johnson, and D.J. Prideaux, "ProblemBased Learning: Its Rationale.” Australian Medical Journal, Vol. 168, p. 445-448, 1998.

[9] D. Jonassen, J. Strobel, and C. B. Lee, "Everyday problem solving in engineering: Lessons for engineering educators," J. Eng. Educ., vol. 95, no. 2, pp. 139-151, 2006.

[10] C. Major, C., and B. Palmer. Assessing the Effectiveness of Problem-Based Learning in Higher Education: Lessons from the Literature. Academic Exchange Quarterly, Vol. 5, p. 4, 2001. 
[11] D. Gijbels, F. Dochy, P. Van den Bossche and M. Segers, "Effects of problem-based learning: a metaanalysis from the angle of assessment" Review of Educational Research, vol. 75, no. 27, 2005.

[12] B.J. Reiser, "Scaffolding complex learning: the mechanisms of structuring and problematizing student work." The Journal of the Learning Sciences, vol. 13, no. 3, p. 273-304, 2004.

[13] D. Jonassen, "Supporting problem solving in PBL," Interdiscipl J. Problem Based Learn., vol. 5, no. 2, p. 95-112, 2011.

[14] H.G. Schmidt, S.M. Loyens, T. Van Gog, and F. Paas, "Problem-based learning is compatible with human cognitive architecture: commentary on Kirschner, Sweller, and Clark," Educational Psychologist, vol. 42, no. 2, p. 91-97, 2007.
[15] H.G. Schmidt, J.I. Rotgans, and E.H.J. Yew, "The process of problem-based learning: what works and why," Medical Education, vol. 45, p. 792-806, 2011.

[16] R.M. Clark and A. Mahboobin, "Scaffolding to support problem-solving performance in a bioengineering lab - a case study", IEEE Transactions on Education, vol. 61, no. 2, 2018.

[17] B. Frank, N. Simper, J. Kaupp, "Formative feedback and scaffolding for developing complex problem solving and modelling outcomes," Eur. J. Eng. Educ., p. 1-17, 2017.

[18]Epstein Educational Enterprises, Immediate Feedback Assessment Technique, http://www.epsteineducation.com/home/about/ 